Introduction We previously reported that our practice of screening for and prevention of opportunistic infections (OI's) in at risk IBD patients was not in line with ECCO recommendations. ${ }^{12}$ In this study, we aimed first to seek evidence of OI's in our historical cohort of infliximab (IFX)-treated patients who had not been screened according to recommendations and second, to analyse the cost-effectiveness of screening of, and prophylaxis as required for tuberculosis (TB) and hepatitis B (hep B) in IFX-treated patients.

Methods An audit of consecutive patients treated with IFX (2005-2009) was conducted and occurrence of OIs and duration of IFX were recorded. A cost-minimisation analysis was performed using clinical decision analytic modelling to assess cost outcome of screening and prevention versus not screening and prevention. Model branch probabilities were obtained from a Medline search. The cost perspective was the health sector. Hospital admissions were based on Healthcare Resource Group (HRG4) codes.

Results There were no OI's identified in 92 IBD patients treated with a median of 21 months of IFX. For 1000 hypothetical Crohn's patients in a western European population, the model predicted 15 screened patients required isoniazid prophylaxis, with 0.5 unscreened patients admitted to hospital with TB. There was an incremental cost for screening of $£ 39.50 /$ patient for CXR alone, or $£ 125.60$ for CXR combined with an interferon release assay. Conversely, there was a cost saving for screening in Southern Europe (£12/patient). For Hepatitis B in the Western European population, the model predicted 35.7 screened patients required lamivudine prophylaxis, whereas in the unscreened group, 2 patients reactivated with hepatitis, 3 patients with liver failure, and one required a transplant. There was a cost saving of $£ 32 /$ patient for screening.

Conclusion There were no significant OI's identified in this audit of IFX-treated IBD patients despite failure to screen according to ECCO guidelines. The cost analysis model demonstrates cost saving for hepatitis B screening because of the significant cost of hepatitis $B$ reactivation. In the southern European population there is a cost benefit for $\mathrm{TB}$ screening due to a high rate of latent $\mathrm{TB}$ whereas in the western European population, there is an incremental cost for screening because of a lower risk of TB reactivation there. This cost should be balanced against the risk of death due to TB reactivation (reported in Southern but not Western Europe). ${ }^{3}$

Competing interests None.

\title{
REFERENCES
}

1. Rahier JF, et al. J Crohns Colitis 2009;3:47.

2. Elliott TR, et al. J Crohns Colitis 2010;4:S63.

3. Fonseca JE, et al. Acta Reumatol Port 2006;31:247-53.

\section{PWE-038 COST ANALYSIS OF SCREENING ACCORDING TO ECCO GUIDELINES FOR PREVENTION OF OPPORTUNISTIC INFECTIONS IN INFLIXIMAB- TREATED IBD PATIENTS}

